\title{
A Rare Complication of Endovascular Embolization: Extruded Onyx on Trigeminal Nerve
}

\author{
Pinar Eser Ocak ${ }^{1}$ Mustafa Kemal Başkaya ${ }^{1}$ \\ ${ }^{1}$ Department of Neurological Surgery, University of Wisconsin School of \\ Medicine and Public Health, Madison, Wisconsin, United States \\ J Neurol Surg B 2018;79(suppl S5):S422-S423.
}

\begin{abstract}
Address for correspondence Mustafa Kemal Başkaya, MD, Department of Neurological Surgery, University of Wisconsin School of Medicine and Public Health, 600 Highland Avenue, K4/8 CSC, P.O. Box 8660, Madison, WI 53792, United States (e-mail: baskaya@neurosurgery.wisc.edu).
\end{abstract}

\begin{abstract}
Keywords

- cerebellopontine angle

- onyx

- embolization

- trigeminal nerve

- hemangioblastoma

Objectives To demonstrate a rare complication of endovascular tumor embolization with onyx.

Design Operative video.

Setting Department of neurological surgery in a university hospital.

Participants A39-year-old male who was diagnosed with a right sided hemangioblastoma.

Main Outcome Measures Surgical resection of the tumor, preservation of the cranial nerves and extruded embolization material on trigeminal nerve.

Results The tumor was embolized with onyx the day before surgery. Patient woke up with no sensation in the right side of his face. Diffusion magnetic resonance imaging (MRI) showed a small restricted diffusion area within the right superior cerebellar vermis. Microsurgical resection of the tumor was uneventful and complete resection was achieved (Fig. 1). After the resection was completed, the trigeminal nerve was identified. Some of the capillaries overlying the nerve as well as the cerebellum and brain stem had extruded onyxembolic material (Fig. 2). Some of the onyx over the cerebellum was removed; however, the ones on the trigeminal nerve and brain stem were not removed due to the risk of injury to the nerve. Postoperative MRI confirmed total resection. Patient made excellent recovery except he continued to have no sensation in the right side of his face.

Conclusion Preoperative embolization is an important adjunct to resection of large hemangioblastomas in selected cases because it may facilitate circumferential dissection and debulking of the tumor. Although extrusion of the embolization material is relatively common, immediate extrusion of onyx and its transfixion on a cranial nerve has not been reported before. Judicial selection of preoperative embolization is required in hemangioblastomas.

The link to the Video can be found at: https://youtu.be/s0DjD26Xkas.
\end{abstract}

Conflict of Interest

None.

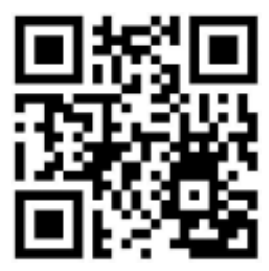

received

May 31, 2018

accepted

August 12, 2018

published online

September 25, 2018 www.thieme.com/skullbasevideos

www.thieme.com/jnlsbvideos

DOI https://doi.org/

10.1055/s-0038-1669972.

(c) 2018 Georg Thieme Verlag KG

Stuttgart · New York
License terms

c) $(1) \$$ 


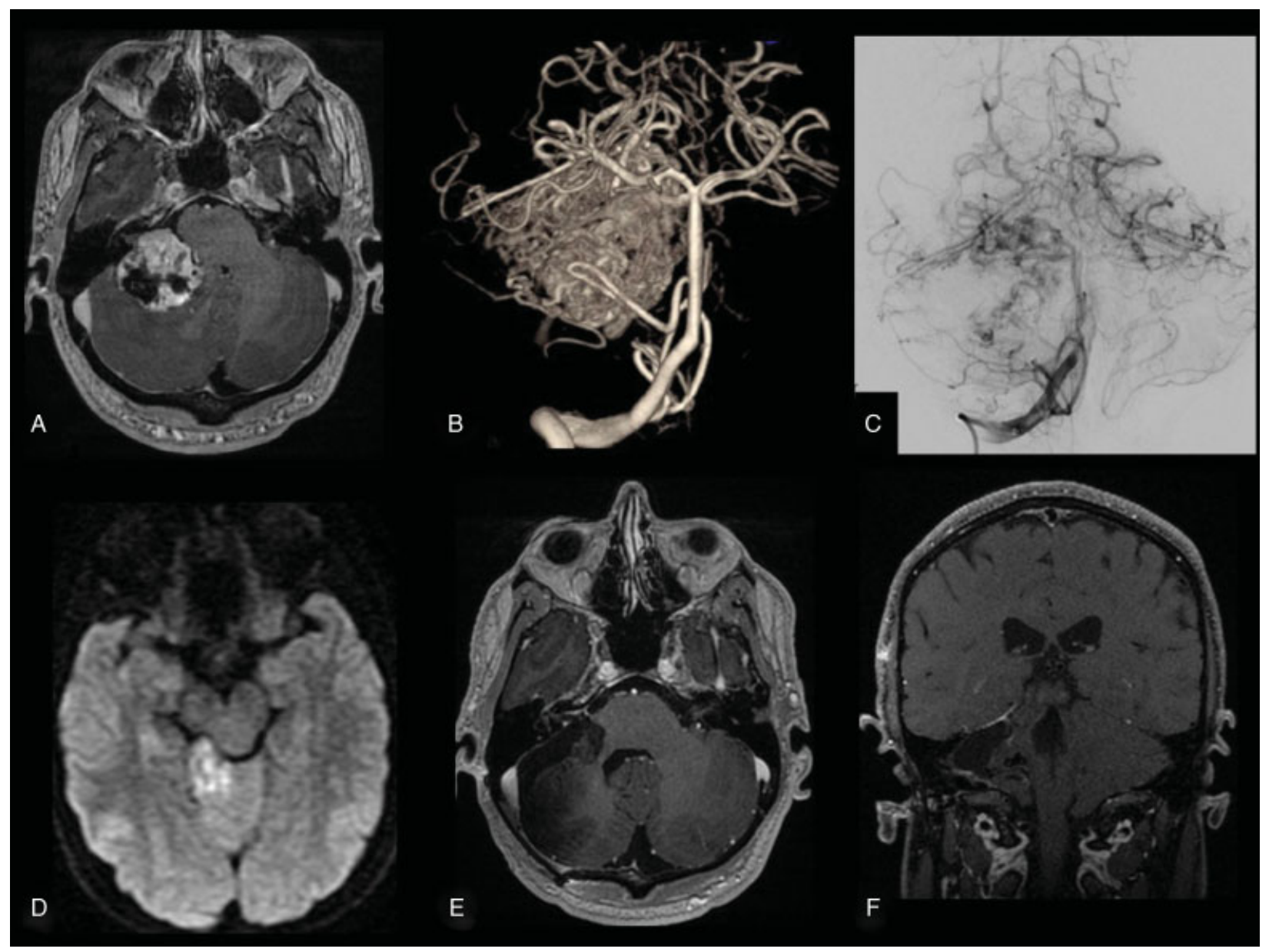

Fig. 1 (A) Preoperative T1-weighted postcontrast axial MR images of a 39-year-old man demonstrating heterogeneously enhancing posterior fossa mass centered at the right cerebellopontine angle with significant vasogenic edema. (B) 3D reconstruction of preembolization angiogram. (C) Postembolization angiogram showing embolized portions of the tumor. (D) Diffusion weighted MR images obtained after endovascular onyx embolization showing a restricted diffusion area in the right superior cerebellar vermis. (E, F) Postoperative T1-weighted postcontrast axial and coronal MR images demonstrating gross total resection of the tumor. 3D, three dimensional; MR, magnetic resonance;

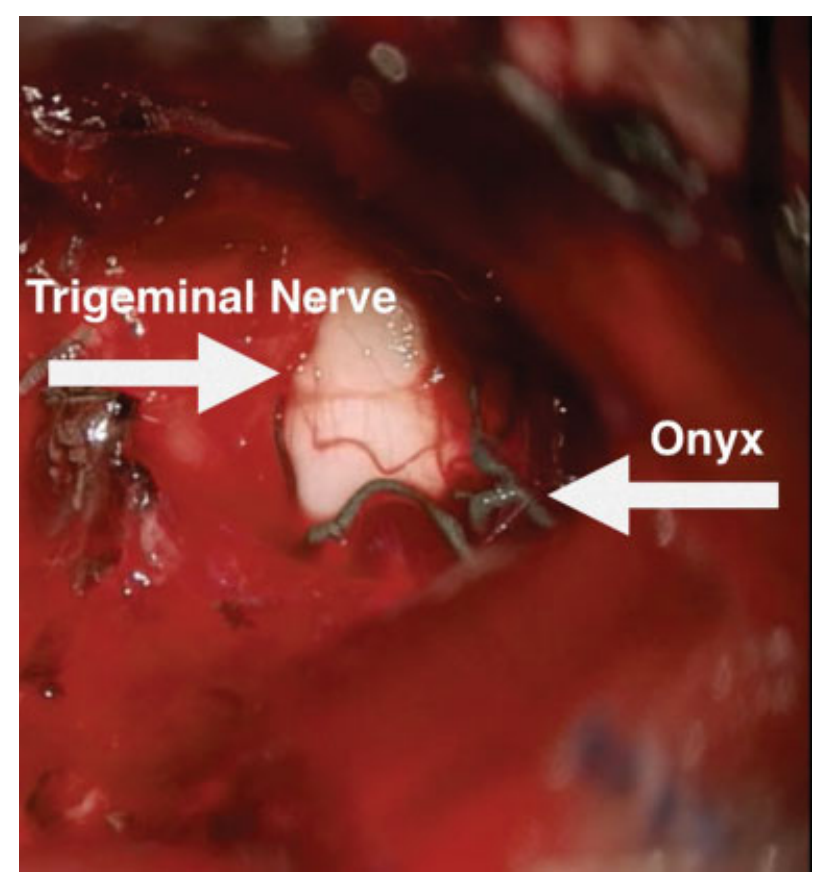

Fig. 2 Intraoperative microscopic image demonstrating the extruded onyx and its penetration to the trigeminal nerve. 